\title{
Insulator Defect Detection Technology Research
}

\author{
Yang Gao ${ }^{1, a^{*}}$, Aoran Xu' ${ }^{1, b}$, Qun Ouyang ${ }^{2, c}$, \\ Liu Zhang ${ }^{1, d}$, Yupeng Wang ${ }^{3, e}$ \\ ${ }^{1}$ Shenyang Institute of Engineering, Shenyang, Liaoning Province, china \\ ${ }^{2}$ State Grid Liaoning Electric Power Company, Shenyang, Liaoning Province, china \\ ${ }^{3}$ State Grid Fuxin Electric Power Supply Company, Fuxin, Liaoning Province, china

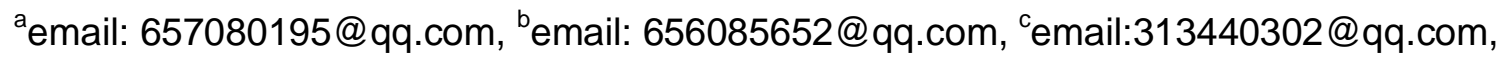 \\ demail: 13918678@qq.com, eemail: 451932741@qq.com
}

Keywords: synthetic insulator; charged detection; ultraviolet pulse; electric field method.

Abstract: Nonlinear composite insulator voltage distribution is big, the umbrella skirt interval is small, this brought difficulty to defect inspection of composite insulator. Article is studied based on electric power within the composite insulator type defect inspection technology, the principle of uv pulse method, ultrasonic method and electric field method demonstrates in detail; Studied based on the contact type and non-contact composite insulator defect in charged detection technology, and made comprehensive comparison table for non-contact; Within the composite insulator is studied based on line defect charged detection technology, and map the working principle of the infrared thermal image; Technology of electric insulator in defect inspection technology outlook.

\section{INTRODUCTION}

Synthetic insulator due to its special electromagnetic field and the natural environment, the need to periodically troubleshoot and repair. Currently the primary means for the detection of charged synthetic insulator is visual inspection and using spray methods hydrophobic insulator grading. The vast majority of composite insulator in the high-altitude high-pressure work environment, how simple, practical and effective way to detect power system robustness composite insulator, has become an important topic of joint research scholars ${ }^{[1-3]}$.

In recent years, the speed of technological development Insulator detected quickly, especially on synthetic insulators since the advent of organic material, because of its outstanding stain resistance and light weight, easy maintenance, cost-effective series of advantages, access to rapid development. Due to the impact of meteorological disasters and years, hidden in the synthesis of the insulator is not easy to find internal defects ${ }^{[4-5]}$, so in order to promptly detect internal defects composite insulator, preventing the line accident inconvenience, synthetic insulators running live detection has important practical significance.

\section{SYNTHESIS RESEARCH STATUS CHARGED INSULATOR DEFECT DETECTION TECHNOLOGY}

Based on internal battery charged type composite insulator defect detection technology can be divided into non-battery detection and power detection.

Non-power detection mainly including ultraviolet pulse method, creeping wave method, 
visual method; electricity including electric field, pulse current method, the distribution voltage method. Currently more popular and simple and practical mainly ultraviolet pulse method, ultrasonic testing method and the electric field method.

(1) UV Pulse Method

When the high-voltage electrical equipment corona discharge, discharge spectroscopy and applied voltage are related. This phenomenon can lead to non-uniform field strength sharp uneven distribution voltage, which turn into partial discharge insulator surface, the electric field around the - board discharge physics model to simulate. Different applied voltages tip - Spectroscopy corona discharge plate shown in Fig2.

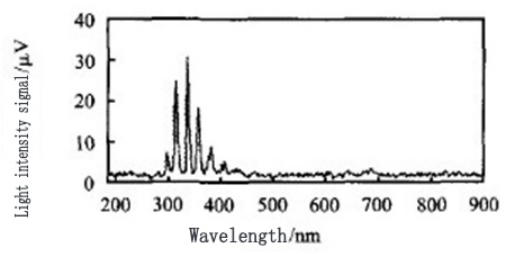

(a) $\mathrm{U}=4.3 \mathrm{KV}$ spectra when

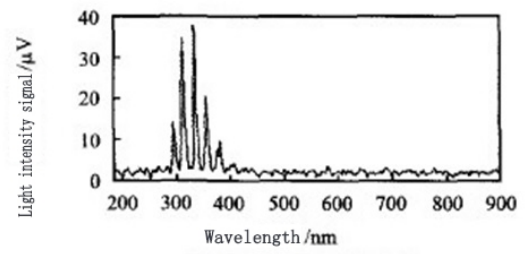

(b) $\mathrm{U}=5 \mathrm{KV}$ spectra when

Figure 1 different voltages tip - plate discharge corona discharge spectrum

Figure 1 shows the corona discharge near ultraviolet spectrum comprises $(100-400 \mathrm{~nm})$, visible light $(400 \sim 760 \mathrm{~nm})$, infrared $(770-1 \mathrm{~mm}) 3$ spectral bands, both continuous spectrum, there are bands and the separation line, This means that the spectrum of the corona discharge from the emission spectrum of molecular spectroscopy, atomic and ionic composition. Figure 2 shows that, when the applied voltage transition from $4.3 \mathrm{KV}$ to $5 \mathrm{KV}$, spectrum ultraviolet pulse signal has been greatly increased. The literature shows that IR and UV exactly the opposite, and visible light is generally not sensitive to voltage changes. This just shows the ultraviolet pulse voltage can characterize the size and discharge strength, so as to detect ultraviolet light pulse signal and discharging high-voltage equipment basis.

UV wavelength in the range 100-400nm, Figure 2 shows a typical corona discharge mainly $300-400 \mathrm{~nm}$ spectral bands of the ultraviolet region, there is a small portion of wavelengths 230-280nm. UV is part of sunlight, but Ozone can absorb wavelengths below $280 \mathrm{~nm}$ light waves, so can atmospheric transport of only a $400 \mathrm{~nm}$ UV $280 \mathrm{~nm}$ signal can penetrate the atmosphere, due to the less than $280 \mathrm{~nm}$ light waves will be filtered out, so we It is called a "blind date." If the signal path ultraviolet light detection system of choice in the solar blind ultraviolet corona discharge detection, warning signal, can be an ideal detection results, that synthetic insulators can detect internal flaws charged to provide evidence.

(2) ultrasonic measurement method - creeping wave method

Poor insulator partial discharge is generated when the discharge of the sonic and ultrasonic, spread to the surrounding, it is possible to use an ultrasonic sensor to measure ultrasonic poor insulator issued, according to the intensity of ultrasound to determine the bad insulator. The schematic diagram shown in Figure 2.

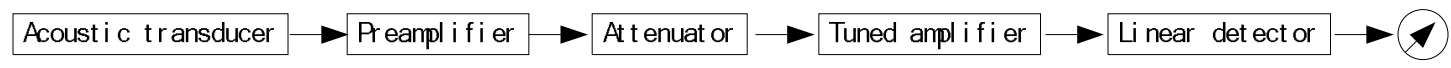

Figure 2 a block diagram schematic of an ultrasonic probe

Ultrasonic measurement method based on testing using the waveform can be divided into longitudinal wave - wave method, creeping wave method (small angle detection), etc., while climbing the law is a very mature and very typical ultrasonic inspection method, the mechanical principle shown in Figure 3 . 
The use of artificial defect on the block as a standard sample testing guidelines, the use of detectors to make distance-amplitude (DAC) curve, as shown in Figure 4. If the ultrasonic wave incident angle of 8 to 150 internal insulator, when detecting the reflected wave above or below the standard DAC curve. If higher DAC curve is considered a defect; if less than DAC curve is considered qualified.

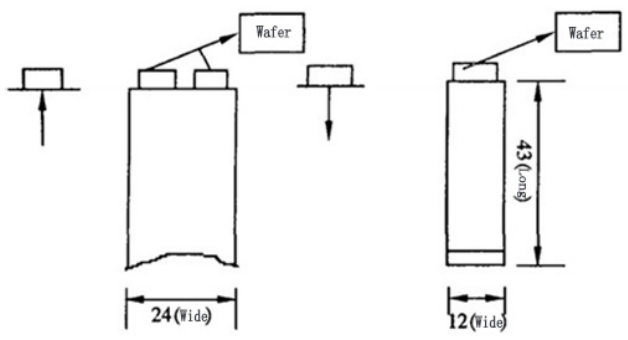

Figure 3 schematic climbing probe

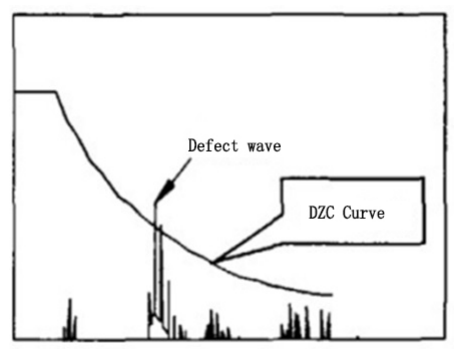

Figure 4 DAC curve

(3) electric field method

Electric field method by detecting the axial direction along the insulator string electric field distribution, and to detect an insulator with a zero value normal electric field distribution. The method does not require contact with the insulator instrument, can make a judgment directly from the farm.

Electric field method can detect hidden internal flaws and external insulation level drop of failure, it can determine the severity of the defect, facilitate composite insulators condition-based maintenance. The literature shows that both AC or DC, good clean insulator strings along the axial direction of the electric field distribution curves are smooth, showing a pattern: high pressure fittings near the end of the maximum field strength, along with the ground moving field strong decreases rapidly near the ground terminal fittings are rising; when the insulator string in insulator or insulator zero value exists in continuity fault in the insulation, where the potential distribution becomes a constant, since the electric field is potential for insulation differential material distance $(E=-\Delta \Phi)$, and therefore the electric field strength at the position corresponding to suddenly decrease, so that the entire distribution of the electric field is no longer a smooth curve. Insulator string electric field distribution curve diagram shown in Fig5.

Figure 5 shows that, when the DC insulator string in internal continuity fault or zero value insulators, electric and harmonic voltage of the DC voltage generated by the electric field generated at the fault will be distorted at present. In theory, within the defect can be charged DC Insulator be detected by measuring the DC insulator DC electric field distribution or a harmonic electric field distribution. 


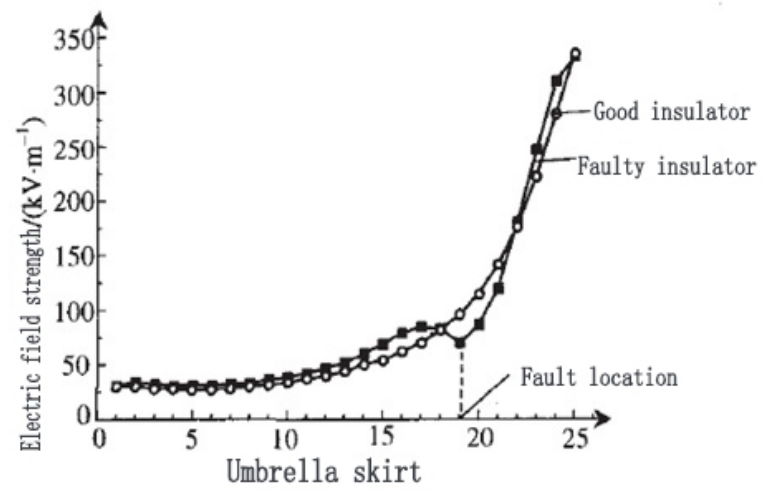

Figure 5 good insulators and insulator electric field distribution curve fault

Based contact method can be divided into non-contact detection and contact detection.

Non-contact detection including ultrasonic testing, laser Doppler vibration, infrared thermometry, Corona camera method and a radio wave detection method. Press contact detection method works mainly electric field method, hydrophobic detection method and leakage current detection method.

Electric field method has been done is described in Section 1.1. I will not elaborate.

Hydrophobic detection methods: the main method currently testing hydrophobic insulator static contact angle method (CA method), dynamic contact angle method, water fractionation (HC method), dynamic drip method.

Wherein the first spray grading by the Swedish Transmission Research Institute (STRI) suggested that the use of HC grade Characterization of Hydrophobic state where the HC1 HC3 is hydrophobic state, HC4 is limbo, HC5 HC7 is a hydrophilic state. Experiments with ordinary watering can to test surface spray mist to observe the distribution of moisture in the surface of the sample, compared to the standard classification criterion and pictures, draw the insulator surface hydrophobic condition, which can determine the insulator insulation. The disadvantage of this method lies in the more dependent on subjective judgments.

Leakage current measurement: for well-insulated insulation, the leakage current and the applied DC voltage should be a linear relationship, when all or part of the defective insulation or damp, the leakage current will increase dramatically, the voltage characteristics were no longer a straight line. Therefore, it can be detected by the test subjects was whether the insulation or moisture, particularly in the discovery of defects inside the insulation, this test more of its special significance.

\section{3 based on in-line type of synthetic insulators charged defect detection technology}

Based on in-line type of synthetic insulators charged defect detection technology can be divided into online and offline testing, currently more common or online testing technology. Line detection technologies include insulator voltage distribution line monitoring photodetector bar method, acoustic pulse detection, corona pulse detection, infrared imaging detection and laser shock detection. Infrared detection is representative of online testing technology..

\section{TECHNICAL OUTLOOK}

Today's internal insulator defect detection technique is to combine live Zigbee wireless networking technology for real-time transmission of measured values of the direction of the electric field of the insulator in different parts of the development, which will be solved after the first measurement to view the measurement results of malpractice, you can generate MLM edge field curve, which greatly improved the efficiency of the detector Insulator. 
(1) The new field measuring probe

Research on electric field measurement technology, high-frequency electromagnetic shielding technology, suspension conductor discharge mechanism and prevention measures, the choice of insulating material designed to connect handle field between the measuring probe and the carriage develop more suitable for field use, simple, flexible, anti-strong field and Better corona resistance of field measurement techniques.

(2) the detection sensitivity of the probe

With this electric field measurement technique to detect the orientation of research in the laboratory, equalizing ring, insulator shape, atmospheric humidity and contamination and other factors on the detection sensitivity.

(3) calibrate field probes

Calculation various angles suspension insulator longitudinal electric field distribution around the research field measuring probe deviation measurement values and actual values, thereby calibrate field probes, gives true value of the electric field distribution around the insulator. Combined with the current latest wireless networking technology for real-time data transmission, improve detection accuracy.

\section{CONCLUSIONS}

The electric field of charged defect detection method is applied to relatively mature, the implementation process within the insulator UV pulse method is simple, but the error is large, climbing law is currently applied only outside defects, internal defect detection, domestic scholars yet to respond.Contacted implement burdensome, but high precision, non-contact is easy to implement, but poor detection.Infrared detection line detection technology because of its good robustness and accuracy, is widely used.

With Zigbee wireless networking technology innovation, the inner insulator charged defect detection technology is to combine Zigbee wireless networking technology direction, in order to achieve real-time transmission of measurement field values in different parts of the insulator, which will become the future of China insulator defect Charged important subject detection technology.

\section{REFERENCE}

[1] Lee Chan roar of a tiger, Niu Ben, had Rong, Yu Zhanqing, Zhang increase detection based on electric field sensors photoelectric composite insulators internal insulation fault [J] High Voltage 2014, (08): 2422-2426.

[2] Luoling, Zhang increase, Ren Guiqing, Liao Yifan, Wang Liming, Li Li + HLJKW feasibility study, zinc horseshoe ring suppression of DC insulators iron cap electrolytic corrosion [J] High Voltage 2014, (07):... 2000-2004.

[3] Amir Syahir, Kotaro Kajikawa, Hisakazu Mihara. Sensitive Detection of Small Molecule-Protein Interactions on a Metal-Insulator-Metal Label-Free Biosensing Platform [J]. Chem. Asian J., 2012, Vol.7 (8) .

[4] SU Zhi-yi impulse breakdown test air line insulator Detection [J]. Power System Technology 2014 (07): 1798-1803.

[5] Ranxue Bin, Cruz, was established, Chen Guo-jun, shut Zhicheng BREAKING Research and degradation mechanism of $500 \mathrm{kV}$ composite insulators Wang Liming analysis, [J] high-voltage electrical 2013, (09): 35-40. 